\title{
LC3, an autophagosome marker, is expressed on oligodendrocytes in Nasu-Hakola disease brains
}

\author{
Jun-ichi Satoh ${ }^{1 *}$, Nobutaka Motohashi ${ }^{2}$, Yoshihiro Kino ${ }^{1}$, Tsuyoshi Ishida ${ }^{3}$, Saburo Yagishita ${ }^{4}$, Kenji Jinnai ${ }^{5}$, \\ Nobutaka Arai ${ }^{6}$, Kiyotaka Nakamagoe ${ }^{7}$, Akira Tamaoka ${ }^{7}$, Yuko Saito ${ }^{8}$ and Kunimasa Arima ${ }^{9}$
}

\begin{abstract}
Background: Nasu-Hakola disease (NHD) is a rare autosomal recessive disorder characterized by sclerosing leukoencephalopathy and multifocal bone cysts, caused by a loss-of-function mutation of either DAP12 or TREM2. TREM2 and DAP12 constitute a receptor/adaptor signaling complex expressed exclusively on osteoclasts, dendritic cells, macrophages, and microglia. Neuropathologically, NHD exhibits profound loss of myelin and accumulation of axonal spheroids, accompanied by intense gliosis accentuated in the white matter of the frontal and temporal lobes. At present, the molecular mechanism responsible for development of leukoencephalopathy in NHD brains remains totally unknown.
\end{abstract}

Methods: By immunohistochemistry, we studied the expression of microtubule-associated protein 1 light chain 3 (LC3), an autophagosome marker, in 5 NHD and 12 control brains.

Results: In all NHD brains, Nogo-A-positive, CNPase-positive oligodendrocytes surviving in the non-demyelinated white matter intensely expressed LC3. They also expressed ubiquitin, ubiquilin-1, and histone deacetylase 6 (HDAC6) but did not express Beclin 1 or sequestosome 1 (p62). Substantial numbers of axonal spheroids were also labeled with LC3 in NHD brains. In contrast, none of oligodendrocytes expressed LC3 in control brains. Furthermore, surviving oligodendrocytes located at the demyelinated lesion edge of multiple sclerosis (MS) did not express LC3, whereas infiltrating Iba1-positive macrophages and microglia intensely expressed LC3 in MS lesions.

Conclusions: These results propose a novel hypothesis that aberrant regulation of autophagy might induce oligodendrogliopathy causative of leukoencephalopathy in NHD brains.

Keywords: Autophagy, LC3, Leukoencephalopathy, Nasu-Hakola disease, Oligodendrocytes

\section{Background}

Nasu-Hakola disease (NHD), also designated polycystic lipomembranous osteodysplasia with sclerosing leukoencephalopathy (PLOSL; OMIM 221770), is a rare autosomal recessive disorder, characterized by progressive presenile dementia and formation of multifocal bone cysts $[1,2]$. Although NHD patients are clustered in Japan and Finland, approximately $200 \mathrm{NHD}$ cases are presently reported worldwide (http://www.orpha.net). Clinically, the patients show pathological bone fractures during the third decade of life, and a frontal lobe syndrome, such as loss of judgment and social inhibitions during the fourth decade

\footnotetext{
*Correspondence: satoj@my-pharm.ac.jp

'Department of Bioinformatics and Molecular Neuropathology, Meiji

Pharmaceutical University, Tokyo, Japan

Full list of author information is available at the end of the article
}

of life, followed by progressive dementia and death until the fifth decade of life [3]. Pathologically, NHD brains exhibit extensive demyelination with sparing of subcortical U-fibers, accumulation of axonal spheroids, and intense astrogliosis predominantly in the white matter of frontal and temporal lobes and the basal ganglia [4]. Genetically, NHD is caused by the set of heterogeneous mutations located in one of the two genes, DNAX-activation protein 12 (DAP12), alternatively named TYRO protein tyrosine kinase-binding protein (TYROBP) on chromosome 19q13.1 or triggering receptor expressed on myeloid cells 2 (TREM2) on chromosome 6p21.1 [5-7]. Previous studies identified 7 different mutations in the TYROBP gene and 11 distinct mutations in the TREM2 gene in NHD patients. The presence of multiple bone cysts, basal ganglia calcification, and genetic mutations of TYROBP or 
TREM2 in a pattern of autosomal recessive inheritance could differentiate NHD from hereditary diffuse leukoencephalopathy with spheroids (HDLS; OMIM 221820), a rare autosomal dominant disorder presenting with clinicopathological similarities to NHD, which is caused by genetic mutations in the colony-stimulating factor 1 receptor (CSF1R) gene [8].

TREM2, expressed exclusively on myeloid cells, such as osteoclasts, dendritic cells, macrophages, and microglia, acts as a receptor for as yet unidentified ligands. TREM2 constitutes a signaling complex with an adaptor molecule DAP12, leading to phosphorylation and activation of the downstream kinase named spleen tyrosine kinase (Syk), following the receptor engagement [9]. Syk transduces a wide range of downstream signals involved in activation of phosphatidylinositol-3 kinase (PI3K), phospholipase C (PLC), protein kinase C (PKC), and mitogen-activated protein kinase (MAPK) [10].

Increasing evidence indicated that a defect in microglial TREM2/DAP12 function plays a central role in the pathogenesis of NHD [11]. However, at present, the molecular mechanism responsible for development of leukoencephalopathy in NHD brains remains totally unknown. DAP12-knockout mice develop osteopetrosis, thalamic hypomyelination, and synaptic degeneration [12], being phenotypically different from osteolytic lesions and sudanophilic leukoencephalopathy found in NHD patients. Several studies showed that oligodendrocytes, along with microglia, express DAP12 [12,13]. However, follow-up studies could not verify oligodendroglial expression of DAP12 [14]. The synaptic function is also altered in DAP12 loss-of-function (K $\Delta 75)$ mice, attributable to reduced expression of AMPA receptor GluR2 subunit and neurotrophin receptor TrkB [15]. Furthermore, the total number of microglia is greatly reduced in the brain of DAP12-deficient and loss-of-function mice [16,17]. These observations suggest that DAP12 signaling pathway plays a key role in development of microglia and maturation of synapses. Knockdown of TREM2 on cultured mouse microglia inhibits phagocytosis of apoptotic neurons, and stimulates production of proinflammatory cytokines, such as TNF $\alpha$ and IL-1 $\beta$, suggesting that TREM2 plays a key role in the clearance of dying neural cells by microglia to resolve damage-induced inflammation [18]. In contrast to the suggested role of microglial TREM2 in the pathogenesis of NHD, we recently found that TREM2 is not expressed constitutively on human microglia, and Iba1positive microglia are well preserved in the brains of NHD patients with DAP12 mutations [19].

Macroautophagy, hereafter called as autophagy, constitutes a lysosome-mediated degradation process that controls the quality of cytoplasmic components and organelles $[20,21]$. The process of autophagy involves the complex molecular machinery, composed of more than 30 autophagy-related (Atg) proteins and 50 lysosomal hydrolases. Autophagy is initiated by the formation of double-membrane-bound vesicles named autophagosomes that sequester cytoplasmic material in a non-degenerative compartment, followed by fusion with lysosomes, leading to degradation of the autophagic contents. They provide recycling pools of nutrients and membranes, being essential for maintenance of the cellular homeostasis and renovation. When the cells are exposed to protein-damaging insults, autophagy plays a key role in eliminating protein aggregates and damaged organelles, both of which are resistant to degradation by the ubiquitin-proteasome system (UPS) [20,21]. Mice defective in autophagy show severe neurodegeneration accompanied by an accumulation of ubiquitinated protein aggregates [22]. Furthermore, abnormal regulation of autophagy plays a central role in the pathogenesis of human neurodegenerative diseases, such as Alzheimer's disease $(\mathrm{AD})$ and Parkinson's disease (PD), accompanied by neuronal accumulation of insoluble protein aggregates [23].

Because TREM2 serves as a phagocytic receptor of apoptotic neurons [18,24], and the efficient clearance of dead cells requires microtubule-associated protein 1 light chain 3A (LC3)-associated phagocytosis [25], we attempted to study the expression of LC3 in NHD brains by immunohistochemistry. Unexpectedly, we found that LC3 expression is enhanced on oligodendrocytes in NHD brains but not in control brains.

\section{Methods}

\section{Human brain tissues}

Formalin-fixed paraffin-embedded brain tissues of the cerebral cortex, the hippocampus, and the basal ganglia derived from NHD and non-NHD cases were obtained from the Research Resource Network (RRN), Japan. Written informed consent was taken in all the cases at autopsy, following the regulation of the institutional ethics committees. The present study includes five NHD patients, composed of a 42-year-old man (NHD1), a 48-year-old woman (NHD2), a 44-year-old man (NHD3), a 32-year-old woman (NHD4), and a 38-year-old man (NHD5), four neuropsychiatric disease controls affected with myotonic dystrophy (MD), composed of a 68-yearold man (MD1), a 61-year-old man (MD2), a 60-year-old man (MD3), and a 53-year-old woman (MD4), four demyelinating disease controls affected with chronic progressive multiple sclerosis (MS), composed of a 29-year-old woman (MS1), a 40-year-old woman (MS2), a 43-year-old woman (MS3), and a 33-year-old man (MS4), and four subjects who died of non-neurological causes (NC), composed of a 63-year-old man who died of prostate cancer and acute myocardial infarction ( $\mathrm{NC1})$, a 67-year-old man who died of dissecting aortic aneurysm (NC2), a 57-year-old man who died of alcoholic liver 
cirrhosis (NC3), and a 61-year-old man who died of rheumatoid arthritis with interstitial pneumonia (NC4). The homozygous mutation of a single base deletion of $141 \mathrm{G}$ (141delG) in exon 3 of DAP12 was identified in NHD1, NHD2, and NHD5 $[19,26]$, while the genetic analysis was not performed in NHD3 [27] or NHD4 [28].

\section{Immunohistochemistry}

After deparaffination, tissue sections were heated in $10 \mathrm{mM}$ citrate sodium buffer, $\mathrm{pH} 6.0$ or 9.0 by autoclave at $110^{\circ} \mathrm{C}$ for $15 \mathrm{~min}$ in a temperature-controlled pressure chamber (Biocare Medical, Concord, CA, USA). They were treated at room temperature (RT) for $15 \mathrm{~min}$ with $3 \%$ hydrogen peroxide-containing methanol to block the endogenous peroxidase activity. They were then incubated with phosphate-buffered saline (PBS) containing 10\% normal goat or rabbit serum at RT for $15 \mathrm{~min}$ to block non-specific staining, followed by incubation in a moist chamber at $4^{\circ} \mathrm{C}$ overnight with the primary antibodies listed in Table 1. We selected Nogo-A as the most reliable marker highly specific for oligodendrocytes in human brain tissue sections, as reported previously [29]. After washing with PBS, the tissue sections were incubated at RT for 30 min with horseradish peroxidase (HRP)-conjugated secondary antibodies (Nichirei,
Tokyo, Japan), followed by incubation with diaminobenzidine tetrahydrochloride (DAB) substrate (Vector, Burlingame, CA, USA). They were processed for a counterstain with hematoxylin. Negative controls underwent all the steps except for exposure to primary antibody.

\section{Western blot analysis}

To prepare total protein extract, the cells were homogenized in the mammalian protein extraction reagent (M-PER; Thermo Scientific, Rockford, IL, USA) supplemented with a cocktail of protease inhibitors (Sigma, St. Louis, MO, USA). The protein extract was centrifuged at 12,000 rpm for $5 \mathrm{~min}$ at RT, separated on a $15 \%$ SDS-PAGE gel, and transferred onto nitrocellulose membranes. They were labeled at RT overnight with rabbit anti-LC3 antibody (PM036; MBL International, Woburn, MA, USA) that react with MAP1LC3A/B/C or goat anti-heat shock protein HSP60 antibody (sc-1052, N-20; Santa Cruz Biotechnology, Santa Cruz, CA, USA) to standardize protein loading. Then, the membranes were incubated at RT for $60 \mathrm{~min}$ with HRPconjugated anti-rabbit or anti-goat IgG (Santa Cruz Biotechnology). The specific reaction was visualized by exposing the membranes to a chemiluminescent substrate (Thermo Scientific).

Table 1 Primary antibodies utilized for immunohistochemistry in the present

\begin{tabular}{|c|c|c|c|c|c|}
\hline Antibody & Supplier & Code (ID) & Origin & Antigen & Concentration \\
\hline LC3 & MBL & PM036 & rabbit & $\begin{array}{l}\text { recombinant human } L C 3 B \text { spanning amino } \\
\text { acid residues } 1-120 \text { aa }\end{array}$ & diluted at 1: 5000 \\
\hline BECN1 & AnaSpec & 54229 & rabbit & a peptide mapping near the $\mathrm{N}$-terminus of human Beclin-1 & $0.2 \mu \mathrm{g} / \mathrm{ml}$ \\
\hline NBR1 & ProteinTech & 16004-1-AP & rabbit & recombinant human NBR1-6xHis fusion protein & $0.26 \mu \mathrm{g} / \mathrm{ml}$ \\
\hline HDAC6 & Santa Cruz Biotechnology & SC-11420 & rabbit & $\begin{array}{c}\text { a peptide spanning amino acid residues 916-1215 } \\
\text { of human HDAC6 }\end{array}$ & $0.8 \mu \mathrm{g} / \mathrm{ml}$ \\
\hline p62/SQSTM1 & BD Bioscience & 610832 & mouse & $\begin{array}{c}\text { a peptide spanning amino acid residues } 257-437 \\
\text { of human p62 }\end{array}$ & $1 \mu \mathrm{g} / \mathrm{ml}$ \\
\hline Ubiquitin & Dako & Z0458 & rabbit & ubiquitin isolated from bovine erythrocytes & $0.25 \mu \mathrm{g} / \mathrm{ml}$ \\
\hline UBQLN1 & Santa Cruz Biotechnology & sc-14652 & goat & $\begin{array}{l}\text { a peptide mapping within an internal region of } \\
\text { human ubiquilin-1 }\end{array}$ & $1 \mu \mathrm{g} / \mathrm{ml}$ \\
\hline Nogo-A & Santa Cruz Biotechnology & $\mathrm{H}-300$ & rabbit & $\begin{array}{c}\text { a peptide mapping amino acids } 700-1000 \\
\text { of human Nogo-A }\end{array}$ & $0.1 \mu \mathrm{g} / \mathrm{ml}$ \\
\hline MBP & Dako & N1564 & rabbit & MBP purified from human brain & prediluted \\
\hline CNPase & Sigma & $11-5 B$ & mouse & purified human CNPase & ascites fluid 1:500 \\
\hline |ba1 & Wako & 019-19741 & rabbit & $\begin{array}{l}\text { a synthetic peptide corresponding to the } \\
\text { C-terminus of Ibal }\end{array}$ & $0.5 \mu \mathrm{g} / \mathrm{ml}$ \\
\hline GFAP & Dako & N1506 & rabbit & GFAP purified from bovine spinal cord & prediluted \\
\hline NF & Nichirei & 412551 (2 F11) & mouse & NF purified from human brain & prediluted \\
\hline Cleaved CASP3 & Cell Signaling Technology & \#9661 (Asp175) & rabbit & $\begin{array}{l}\text { a peptide mapping amino-terminal residues adjacent } \\
\text { to Asp175 of human caspase- } 3\end{array}$ & 1:100 \\
\hline
\end{tabular}

Abbreviations: LC3, microtubule-associated protein 1 light chain 3; BECN1, Beclin 1; NBR1, neighbor of BRCA1 gene 1; HDAC6, histone deacetylase 6; SQSTM1, sequestosome 1; UBQLN1, ubiquilin-1; MBP, myelin basic protein; CNPase, 2',3'-cyclic nucleotide 3' phosphodiesterase; GFAP, glial fibrillary acidic protein; NF, neurofilament protein; and CASP3, caspase-3. 


\section{Results}

Oligodendrocytes surviving in the non-demyelinated white matter of NHD brains intensely expressed LC3 immunoreactivity

First, we validated the specificity of anti-LC3 antibody PM036 by western blot analysis of total protein extracted from mouse oligodendrocyte-type 2 astrocyte $(\mathrm{O} 2 \mathrm{~A})$ progenitor cells termed OS3 [30], following a 48-hour exposure to rapamycin, a potent inducer of autophagy. This antibody reacted with both LC3-I, the soluble cytosolic form and LC3-II, the autophagy-inducible phosphatidylethanolamine (PE)-conjugated form (Additional file 1: Figure S1a, b, lanes 1, 2). Then, we studied the expression of LC3 in the serial brain sections of five NHD, four MD, and four NC cases by immunohistochemistry using the PM036 antibody. In all cases examined, substantial populations of cortical neurons constitutively expressed LC3 in the cytoplasm at varying intensities. Notably, in all five NHD brains, Nogo-A-positive, cleaved caspase-3
(CASP3)-negative (non-apoptotic) oligodendrocytes surviving in the MBP-positive (non-demyelinated) white matter intensely expressed LC3 with the location in the cytoplasm (Figure 1a-c, Figure 2a-c; Additional file 2: Figure S2a, b). Some LC3-immunolabeled oligodendrocytes showed a morphological feature of swollen cytoplasm (Figure 1d). In contrast, extensively demyelinated white matter, almost totally devoid of oligodendrocytes, was not labeled with anti-LC3 antibody (Figure 1a-c, upper half). Double labeling verified that LC3-expressing cells accumulated in the non-demyelinated white matter of NHD brains coexpressed 2'3'-cyclic nucleotide 3' phosphodiesterase (CNPase), a cell type-specific marker of oligodendrocytes (Figure 2f). The distribution of LC3 immunoreactivity (Figure 2a) was well consistent with the staining pattern of Nogo-A (Figure $2 b$ ) but not of GFAP (Figure 2d) or Iba1 (Figure 2e), although some populations of ramified microglia, accumulating macrophages, and reactive astrocytes expressed intensely LC3 in NHD brains
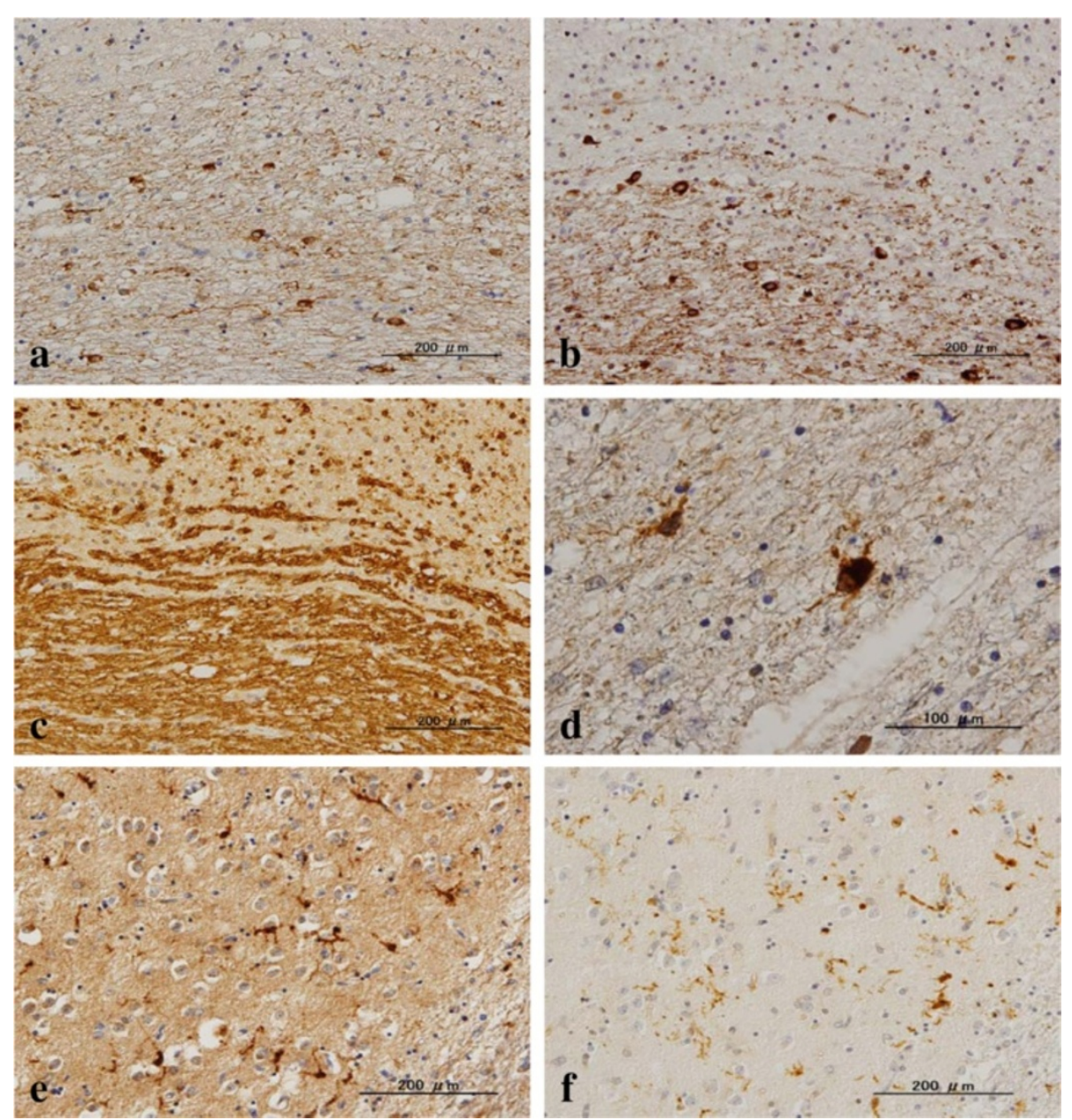

Figure 1 Surviving oligodendrocytes express LC3 in NHD brains. The serial brain sections of NHD cases were processed for immunohistochemistry. The panels (a-f) represent (a) the periventricular white matter, LC3, (b) the same field as (a), Nogo-A, (c) the same field as (a), MBP, the upper half indicates demyelinated lesions, (d) the frontal white matter, LC3, (e) the basal ganglia, LC3, and (f) the same field as (e), Iba1. 

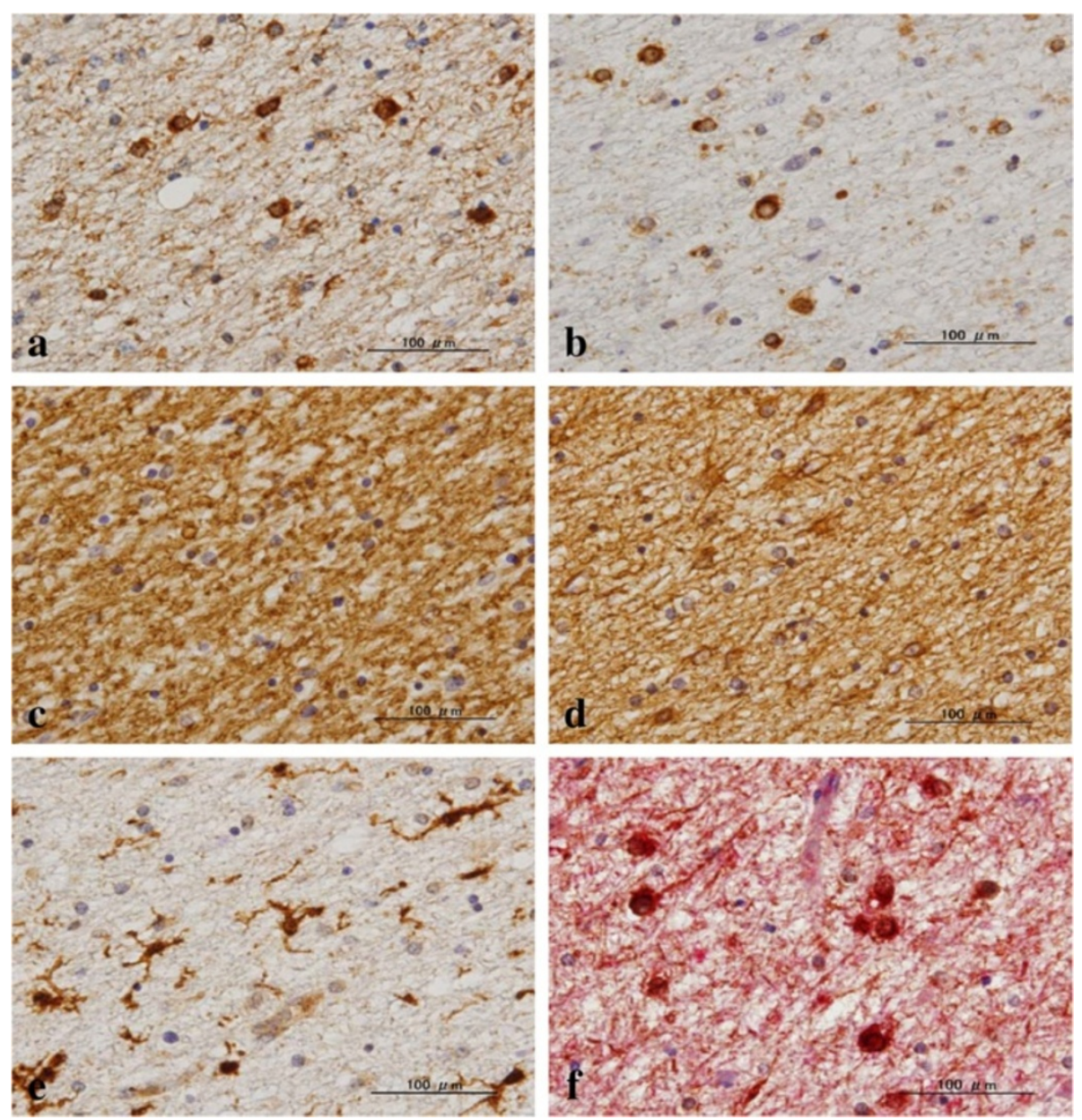

Figure 2 Surviving oligodendrocytes express LC3 in NHD brains. The serial brain sections of NHD cases were processed for immunohistochemistry. The panels (a-f) represent the identical field of the frontal white matter labeled with (a) LC3, (b) Nogo-A, (c) MBP, (d) GFAP, (e) Ibal, and (f) CNPase (red) and LC3 (brown).

(Figure 1e, f). In NHD brains, substantial numbers of axonal spheroids were also labeled with LC3, along with neurofilament (Figure 3a, b). In contrast, we found no LC3-expressing oligodendrocytes in the white matter of control brains, including NC and MD cases (Figure 3c-f).

\section{LC3-positive oligodendrocytes did not express p62 or beclin 1 in NHD brains}

Next, we studied the expression of a panel of autophagy regulators, such as Beclin 1 (ATG6), p62 or NBR1 in NHD brains. Nogo-A-positive LC3-positive oligodendrocytes did not express either Beclin 1 or p62 (Figure 4a-d), although a subpopulation of LC3-positive oligodendrocytes fairly weakly expressed neighbor of BRCA1 gene 1 (NBR1) (Additional file 2: Figure S2c). In contrast, the majority of LC3-positive oligodendrocytes intensely or moderately expressed histone deacetylase 6 (HDAC6) in the cytoplasm (Additional file 2: Figure S2d). Furthermore, many LC3-positive oligodendrocytes moderately expressed both ubiquitin and ubiquilin-1 (UBQLN1) (Figure 4e, f). These observations suggest that the expression of UPS components is upregulated in surviving oligodendrocytes distributed in the non-demyelinating white matter of NHD brains.

\section{Oligodendrocytes surviving at the demyelinated lesion edge in MS brains did not express LC3}

Finally, to investigate whether oligodendroglial LC3 expression represents a general biological process during demyelination, we studied the expression of LC3 in the cerebral white matter of the brains derived from four MS patients. At the edge of chronic active demyelinated lesions, surviving Nogo-A-positive oligodendrocytes did not express LC3 (Figure 5a, b), whereas infiltrating Iba1positive macrophages and microglia intensely expressed LC3 in MS lesions (Figure 5e-f). Furthermore, none of Nogo-A-positive oligodendrocytes expressed LC3 in earlier lesions as well as normal-appearing white matter 

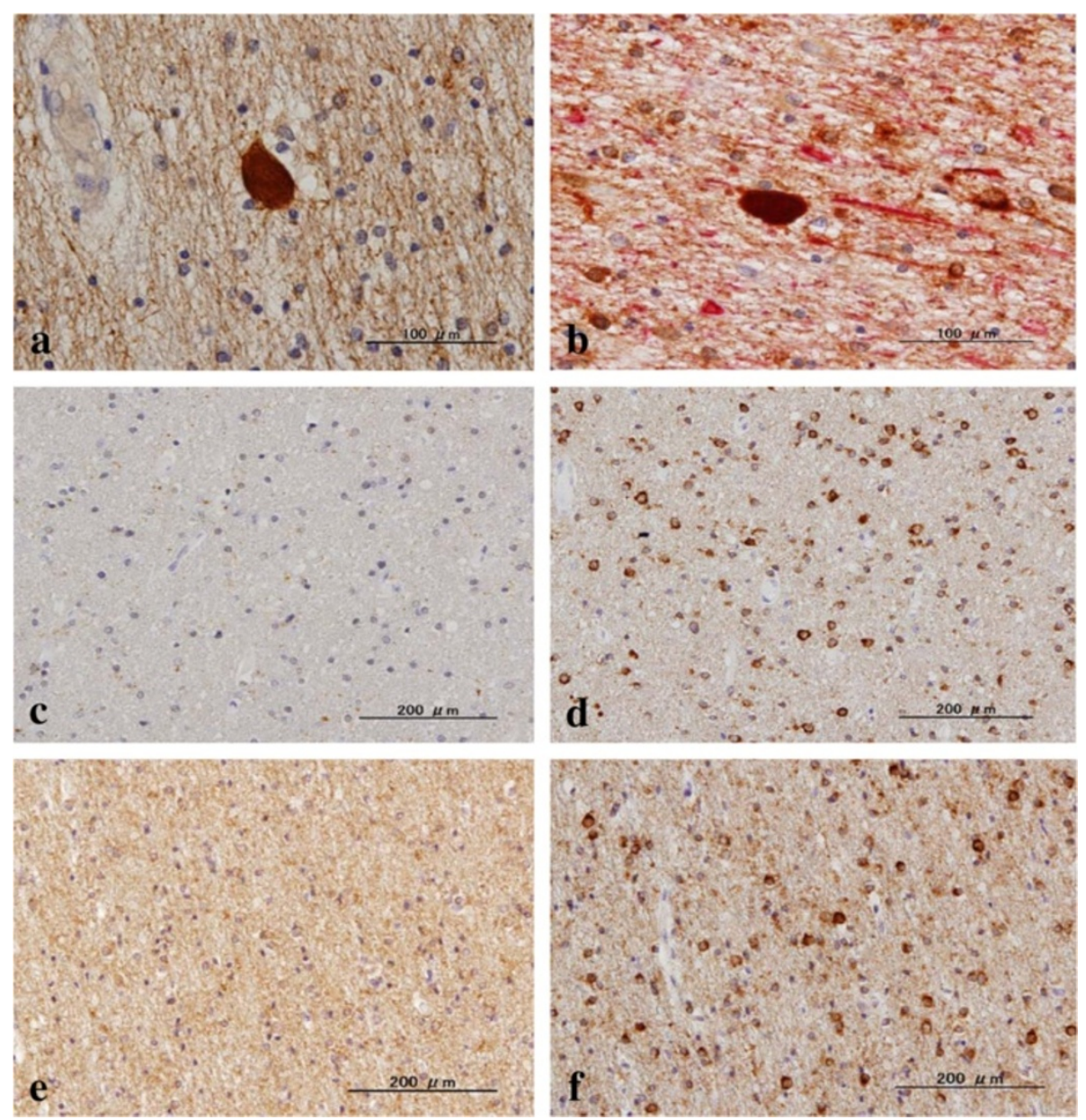

Figure 3 Oligodendrocytes do not express LC3 in control brains. The serial brain sections of NHD, myotonic dystrophy (MD), and non-neurological control (NC) cases were processed for immunohistochemistry. The panels (a-f) represent (a) NHD, the basal ganglia, LC3, (b) NHD, the frontal white matter, neurofilament protein (red) and LC3 (brown), (c) NC, the frontal white matter, LC3, (d) the same field as (c), Nogo-A, (e) MD, the frontal white matter, LC3, and (f) the same field as (e), Nogo-A.

(NAWM) of MS brains (Additional file 3: Figure S3a, b). These observations suggest that oligodendroglial expression of LC3 is not unique to demyelinating events.

\section{Discussion}

Here, we found that oligodendrocytes surviving in the non-demyelinated white matter of NHD brains but not in the white matter of control brains intensely expressed LC3, the most reliable in vivo marker of autophagosomes. LC3-positive oligodendrocytes also expressed ubiquitin, ubiqulin-1 and HDAC6, whereas they marginally expressed NBR1 and did not express Beclin 1 or p62. Furthermore, a subset of axonal spheroids expressed LC3 in NHD brains. Since oligodendrocytes support axonal function by continuously supplying energy metabolites to axons [31], a functional relationship might exist between LC3-expressing oligodendrocytes and axonal spheroids. In contrast, surviving oligodendrocytes located at the demyelinated lesion edge of MS did not express LC3, indicating that different molecular mechanisms might be involved in demyelinating processes between NHD and MS. It is well known that demyelinated lesions in MS brains have a well-demarcated border but the lesions are ill-defined and diffuse in the white matter of NHD brains [4]. Cortical demyelination is common in MS brains [32], while the architecture of the cerebral cortex is well preserved in NHD brains [33]. Demyelinated lesions of MS are often accompanied by perivascular infiltration of numerous $\mathrm{T}$ lymphocytes, while NHD brain lesions contain a limited number of CD3-positive $T$ cells [19], supporting the general view that MS is a T cell-mediated autoimmune disease affecting the central nervous system white matter, whereas autoimmune mechanisms are unlikely to play a central role in the pathogenesis of NHD. Previously, we found that the levels of expression of a guanine nucleotide exchanger for Rap termed RAPGEF4, 

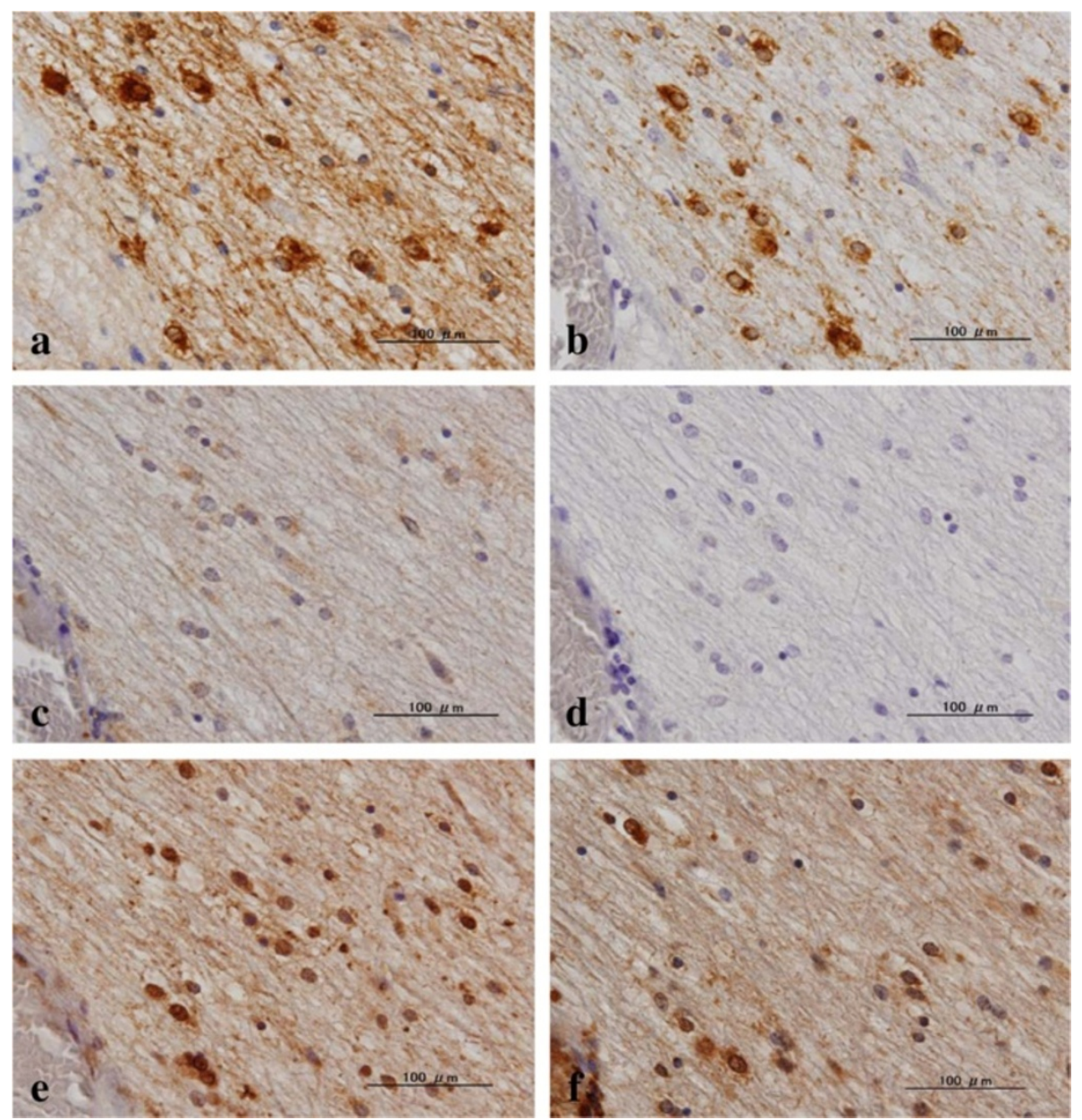

Figure 4 Surviving oligodendrocytes do not express Beclin 1 or p62 in NHD brains. The serial brain sections of NHD cases were processed for immunohistochemistry. The panels (a-f) represent the identical field of the frontal white matter labeled with (a) LC3, (b) Nogo-A, (c) Beclin 1 , (d) p62, (e) ubiquitin, and (f) UBQLN1.

which plays a role in the inhibition of autophagy [34], are greatly reduced in NHD brains [35]. All of these observations suggest the hypothesis that aberrant regulation of autophagy might induce oligodendrogliopathy causative of leukoencephalopathy in NHD brains.

Autophagy is mediated by the molecular machinery that involves numerous regulatory proteins $[20,21]$. Recently, more than 400 interacting proteins that constitute the basal autophagy network have been identified in human cells, representing the extreme complexity of autophagy [36]. LC3 (ATG8), synthesized as a precursor form, is cleaved at its C-terminus by the cysteine protease ATG4B, which generates the cytosolic isoform termed LC3-I [20]. During the phagophore elongation, LC3-I is conjugated to PE via a reaction that involves ATG7 and ATG3 to form LC3-II that is specifically targeted to the elongating autophagosomal membranes. Following the fusion of autophagosomes with lysosomes, LC3-II located on the cytoplasmic face of autolysosomes is delipidated by
ATG4 and processed for recycling, while LC3-II on the internal surface of autophagosomes is processed for degradation by lysosomal enzymes of autolysosomes [20]. All currently available anti-LC3 antibodies, including the PM036 antibody utilized in the present study, recognize both LC3-I and LC3-II. When autophagosomes are accumulated in the cell extremely in number due to excessive induction or reduced completion of autophagy, LC3 intensities are elevated chiefly by an increase in LC3-II expression on autophagosomal membranes.

Under physiological conditions, UPS mainly regulates degradation of short-lived polyubiquitinated proteins, while autophagy predominantly degrades long-lived proteins having higher-ordered structures inaccessible to the narrow pore of the barrel structure of the proteasome, although functionally redundant interactions exist between the two systems [37]. A battery of autophagic receptors/ adaptors that connect the UPS and autophagy, such as p62, NBR1, UBQLN1, optineurin (OPTN), and HDAC6, 

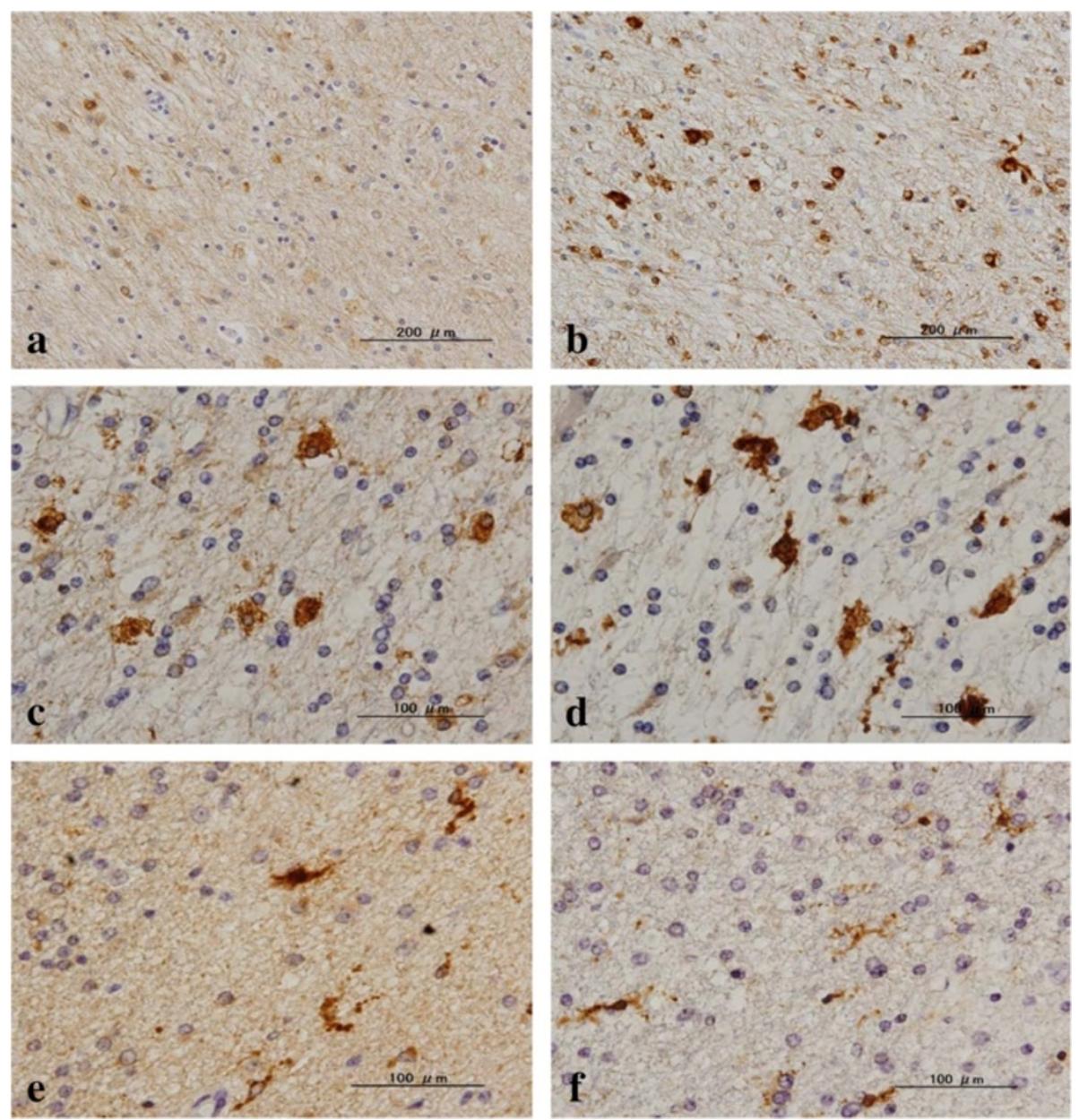

Figure 5 Macrophages and microglia but not oligodendrocytes express LC3 in MS brains. The serial brain sections of multiple sclerosis (MS) cases were processed for immunohistochemistry. The panels (a-f) represent (a) the edge of demyelinated lesions in the parietal white matter, LC3, (b) the same field of (a), Nogo-A, (c) the edge of demyelinated lesions in the frontal white matter, LC3, (d) the same field as (c), Iba1, (e) the edge of demyelinated lesions in the frontal white matter, LC3, and (f) the same field as (e), Iba1.

recognize ubiquitinated target proteins and promote their degradation by autophagy [38]. Importantly, p62, NBR1, UBQLN1, and OPTN have a capacity to bind directly to LC3 $[39,40]$. We found that LC3-positive oligodendrocytes intensely express UBQLN1 and HDAC6, both of which play a pivotal role in the aggresome formation [41,42]. In contrast, LC3-positive oligodendrocytes did not much express either p62 or NBR1. It is worthy to note that p62 knockout mice do not show a defect in bulk autophagy, suggesting that p62 is primarily dispensable for the clearance of autophagic substrates [43].

Under stressful conditions, autophagy serves as a protective mechanism for the cell to prevent the accumulation of cytotoxic protein aggregates and damaged organelles [20,21]. Actually, increased levels of autophagy promote survival of oligodendrocytes in a myelin-deficient rat [44]. However, uncontrolled activation of autophagy often induces cell death. During ischemia-reperfusion injury of the myocardium, early activation of autophagy upon ischemia is protective, while delayed and robust activation of autophagy during reperfusion is detrimental for cell survival [45]. Furthermore, a tight linkage is found between autophagy and apoptosis. The autophagy inhibitor 3-methyladenine (3-MA) inhibits apoptotic cell death of TNF $\alpha$-treated T lymphoblastic leukemia cells and NGFdeprived sympathetic neurons [46,47]. The prototype anti-apoptotic regulator Bcl-2 inhibits starvation-induced autophagy by directly interacting with Beclin 1 [48].

At present, the precise mechanism remains unknown how microglial dysfunction termed microgliopathy caused by the genetic defect of DAP12 or TREM2 induces oligodendrogliopathy characterized by enhanced LC3 expression on oligodendrocytes in NHD brains. It is possible that microglia persistently deregulated in NHD brains produce excessive amounts of reactive oxygen species (ROS) that potentially activate autophagy in 
oligodendrocytes. In turn, autophagy itself controls inflammation through regulatory interactions with innate immune signaling pathways [40]. By gene expression profiling, we recently identified 324. DEGs expressed in frozen brain tissues of a NHD patient with a splicing mutation of TREM2 [35]. Among them, the set of 136 genes involved in inflammatory response and immune cell trafficking are upregulated, while the set of 188 genes including a battery of GABA receptor subunits and synaptic proteins are downregulated in NHD brains. These observations suggested that both neuroinflammatory and neurodegenerative events proceed concurrently in NHD brains. Notably, the expression of a set of macrophage/microglia markers, such as CD163, MSR1, and CD68, is greatly elevated in NHD brains [35].

Upregulation of LC3 is attributable to increased autophagic flux or decreased autophagic substrate clearance, or both. Diverse stress-inducing stimuli, including exposure to ROS and deprivation of nutrients, growth factors, or adenosine triphosphate (ATP), all turns on autophagy by inhibiting the mammalian target of rapamycin complex 1 (mTORC1) [20]. Notably, rapamycin, a relatively selective inhibitor of $\mathrm{mTORC} 1$, ameliorates neurodegeneration in mouse models of $\mathrm{AD}, \mathrm{PD}$, and frontotemporal lobar degeneration (FTLD), where neuronal cell death is attributable to a defect in autophagy $[49,50]$. On the contrary, activation of mTORC1 and mTORC2 is pivotal for oligodendrocytes differentiation at the stage of transition from the late progenitors to immature oligodendrocytes [51]. All of these observations suggest that delicate regulation of cellular autophagy levels plays a decisive role in neural cell survival or cell death.

\section{Conclusions}

We for the first time found that LC3 is expressed on surviving oligodendrocytes in the non-demyelinated white matter of NHD brains but not in the white matter of control brains. These observations propose a novel hypothesis that aberrant regulation of autophagy might induce oligodendrogliopathy causative of leukoencephalopathy in NHD brains.

\section{Additional files}

Additional file 1: Figure S1. Validation of the specificity of anti-LC3 antibody. Total protein extracted from oligodendrocyte-type 2 astrocyte (O2A) progenitor cells named OS3 was processed for western blot with (a) anti-LC3 antibody PM036 and relabeled with (b) anti-HSP60 antibody for standardization of protein loading. The lanes $(1,2)$ indicate a 48 hourtreatment of OS3 cells with (1) the equal $\mathrm{v} / \mathrm{v} \%$ concentration of dimethyl sulfoxide (DMSO) or (2) $1 \mu \mathrm{M}$ rapamycin.

Additional file 2: Figure S2. Surviving oligodendrocytes express HDAC6 in NHD brains. The serial brain sections of NHD cases were processed for immunohistochemistry. The panels (a-d) represent (a) the perivascular white matter, LC3, (b) the same field as (a), cleaved CASP3, (c) the same filed as (a), NBR1, and (d) the same field as (a), HDAC6 with a close-up view in inset.

Additional file 3: Figure S3. Oligodendrocytes do not express LC3 in early lesions of MS brains. The serial brain sections of MS cases were processed for immunohistochemistry. The panels $(a, b)$ represent (a) an early lesion in the frontal white matter, LC3, some macrophages are positive, and (b) the same field as (a), Nogo-A.

\section{Abbreviations}

AD: Alzheimer's disease; CNPase: 2',3'-cyclic nucleotide 3' phosphodiesterase; DAP12: DNAX-activation protein 12; HDAC6: Histone deacetylase 6;

LC3: microtubule-associated protein 1 light chain 3; MD: Myotonic dystrophy; MS: Multiple sclerosis; NBR1: Neighbor of BRCA1 gene 1; NHD: Nasu-Hakola disease; PD: Parkinson's disease; TREM2: Triggering receptor expressed on myeloid cells 2; UPS: Ubiquitin-proteasome system.

\section{Competing interests}

The authors declare that they have no competing interests.

\section{Authors' contributions}

JS and YK performed immunohistochemical analysis. JS drafted the manuscript. NM, SY, KJ, NA, KN, and AT provided NHD brain tissues. KA, YS, and TI validated the pathological diagnosis of all autopsied brains. All authors read and approved the final manuscript.

\section{Acknowledgements}

All autopsied brain samples were obtained from the Research Resource Network (RRN), Japan. This work was supported by grants from the Research on Intractable Diseases, entitled "Clinicopathological and genetic studies of Nasu-Hakola disease" (H21-Nanchi-Ippan-201; H22-Nanchi-Ippan-136), the Ministry of Health, Labour and Welfare of Japan, and the JSPS KAKENHI (C22500322 and C25430054) and the Intractable Disease Research Center (IDRC) project, the Ministry of Education, Culture, Sports, Science and Technology (MEXT), Japan, and the grant from the BioBank of the National Center for Geriatrics and Gerontology (NCGC 26-20).

\section{Author details}

'Department of Bioinformatics and Molecular Neuropathology, Meiji Pharmaceutical University, Tokyo, Japan. ${ }^{2}$ Department of Psychiatry, University of Yamanashi, Faculty of Medicine, Yamanashi, Japan. ${ }^{3}$ Department of Pathology and Laboratory Medicine, Kohnodai Hospital, National Center for Global Health and Medicine, Chiba, Japan. ${ }^{4}$ Department of Pathology, Kanagawa Rehabilitation Center, Kanagawa, Japan. ${ }^{5}$ Department of Neurology, NHO Hyogo-Chuo Hospital, Hyogo, Japan. ${ }^{6}$ Brain Pathology Research Center, Tokyo Metropolitan Institute of Medical Science, Tokyo, Japan. ${ }^{7}$ Department of Neurology, Institute of Clinical Medicine, University of Tsukuba, Ibaraki, Japan. ${ }^{8}$ Department of Laboratory Medicine, National Center Hospital, NCNP, Tokyo, Japan. ${ }^{9}$ Department of Psychiatry, National Center Hospital, NCNP, Tokyo, Japan.

Received: 18 February 2014 Accepted: 17 April 2014

Published: 1 May 2014

\section{References}

1. Nasu T, Tsukahara Y, Terayama K: A lipid metabolic disease - "Membranous lipodystrophy" - an autopsy case demonstrating numerous peculiar membrane-structures composed of compound lipid in bone and bone marrow and various adipose tissues. Acta Pathol Jpn 1973, 23(3):539-558

2. Hakola HP: Neuropsychiatric and genetic aspects of a new hereditary disease characterized by progressive dementia and lipomembranous polycystic osteodysplasia. Acta Psychiatr Scand Suppl 1972, 232:1-173.

3. Bianchin MM, Capella HM, Chaves DL, Steindel M, Grisard EC, Ganev GG, da Silva Júnior JP, Neto Evaldo S, Poffo MA, Walz R, Carlotti Júnior CG, Sakamoto AC: Nasu-Hakola disease (polycystic lipomembranous osteodysplasia with sclerosing leukoencephalopathy - PLOSL): a dementia associated with bone cystic lesions. From clinical to genetic and molecular aspects. Cell Mol Neurobiol 2004, 24(1):1-24.

4. Tanaka J: Nasu-Hakola disease: a review of its leukoencephalopathic and membranolipodystrophic features. Neuropathology 2000, 20(Suppl):S25-S29. 
5. Klünemann HH, Ridha BH, Magy L, Wherrett JR, Hemelsoet DM, Keen RW De Bleecker JL, Rossor MN, Marienhagen J, Klein HE, Peltonen L, Paloneva J: The genetic causes of basal ganglia calcification, dementia, and bone cysts: DAP12 and TREM2. Neurology 2005, 64(9):1502-1507.

6. Paloneva J, Manninen T, Christman G, Hovanes K, Mandelin J, Adolfsson R, Bianchin M, Bird T, Miranda R, Salmaggi A, Tranebjaerg L, Konttinen Y, Peltonen L: Mutations in two genes encoding different subunits of a receptor signaling complex result in an identical disease phenotype. Am J Hum Genet 2002, 71(3):656-662.

7. Kondo T, Takahashi K, Kohara N, Takahashi Y, Hayashi S, Takahashi H, Matsuo H, Yamazaki M, Inoue K, Miyamoto K, Yamamura T: Heterogeneity of presenile dementia with bone cysts (Nasu-Hakola disease): three genetic forms. Neurology 2002, 59(7):1105-1107.

8. Sundal C, Lash J, Aasly J, Øygarden S, Roeber S, Kretzschman H, Garbern JY, Tselis A, Rademakers R, Dickson DW, Broderick D, Wszolek ZK: Hereditary diffuse leukoencephalopathy with axonal spheroids (HDLS): a misdiagnosed disease entity. J Neurol Sci 2012, 314(1-2):130-137.

9. Colonna M: TREMs in the immune system and beyond. Nat Rev Immunol 2003, 3(6):445-453

10. Ivashkiv LB: Cross-regulation of signaling by ITAM-associated receptors. Nat Immunol 2009, 10(4):340-347.

11. Satoh J: Possible role of microgliopathy in the pathogenesis of Nasu-Hakola disease. Clin Exp Neuroimmunol 2013, 4(Suppl 1):17-26.

12. Kaifu T, Nakahara J, Inui M, Mishima K, Momiyama T, Kaji M, Sugahara A, Koito H, Ujike-Asai A, Nakamura A, Kanazawa K, Tan-Takeuchi K, Iwasaki K, Yokoyama WM, Kudo A, Fujiwara M, Asou H, Takai T: Osteopetrosis and thalamic hypomyelinosis with synaptic degeneration in DAP12-deficient mice. J Clin Invest 2003, 111(3):323-332

13. Kiialainen A, Hovanes K, Paloneva J, Kopra O, Peltonen L: Dap12 and Trem2, molecules involved in innate immunity and neurodegeneration, are co-expressed in the CNS. Neurobiol Dis 2005, 18(2):314-322

14. Thrash JC, Torbett BE, Carson MJ: Developmental regulation of TREM2 and DAP12 expression in the murine CNS: implications for Nasu-Hakola disease. Neurochem Res 2009, 34(1):38-45.

15. Roumier A, Béchade C, Poncer JC, Smalla KH, Tomasello E, Vivier E, Gundelfinger ED, Triller A, Bessis A: Impaired synaptic function in the microglial KARAP/DAP12-deficient mouse. J Neurosci 2004 24(50):11421-11428

16. Nataf S, Anginot A, Vuaillat C, Malaval L, Fodil N, Chereul E, Langlois JB, Dumontel C, Cavillon G, Confavreux C, Mazzorana M, Vico L, Belin MF, Vivier $E$, Tomasello $E$, Jurdic P: Brain and bone damage in KARAP/DAP12 loss-of-function mice correlate with alterations in microglia and osteoclast lineages. Am J Pathol 2005, 166(1):275-286.

17. Otero K, Turnbull IR, Poliani PL, Vermi W, Cerutti E, Aoshi T, Tassi I, Takai T, Stanley SL, Miller M, Shaw AS, Colonna M: Macrophage colony-stimulating factor induces the proliferation and survival of macrophages via a pathway involving DAP12 and $\beta$-catenin. Nat Immunol 2009, 10(7):734-743.

18. Takahashi K, Rochford CD, Neumann H: Clearance of apoptotic neurons without inflammation by microglial triggering receptor expressed on myeloid cells-2. J Exp Med 2005, 201(4):647-657.

19. Satoh J, Tabunoki H, Ishida T, Yagishita S, Jinnai K, Futamura N, Kobayashi M, Toyoshima I, Yoshioka T, Enomoto K, Arai N, Arima K: Immunohistochemical characterization of microglia in Nasu-Hakola disease brains. Neuropathology 2011, 31(4):363-375.

20. Ravikumar B, Sarkar S, Davies JE, Futter M, Garcia-Arencibia M, Green-Thompson ZW, Jimenez-Sanchez M, Korolchuk VI, Lichtenberg M, Luo S, Massey DC, Menzies FM, Moreau K, Narayanan U, Renna M, Siddiqi FH Underwood BR, Winslow AR, Rubinsztein DC: Regulation of mammalian autophagy in physiology and pathophysiology. Physiol Rev 2010, 90(4):1383-1435.

21. Mizushima N, Komatsu M: Autophagy: renovation of cells and tissues. Cell 2011, 147(4):728-741.

22. Hara T, Nakamura K, Matsui M, Yamamoto A, Nakahara Y, Suzuki-Migishima R, Yokoyama M, Mishima K, Saito I, Okano H, Mizushima N: Suppression of basal autophagy in neural cells causes neurodegenerative disease in mice. Nature 2006, 441(7095):885-889.

23. Nixon RA: The role of autophagy in neurodegenerative disease. Nat Med 2013, 19(8):983-997.

24. Hsieh CL, Koike M, Spusta SC, Niemi EC, Yenari M, Nakamura MC, Seaman WE: A role for TREM2 ligands in the phagocytosis of apoptotic neuronal cells by microglia. J Neurochem 2009, 109(4):1144-1156.
25. Martinez J, Almendinger J, Oberst A, Ness R, Dillon CP, Fitzgerald P, Hengartner MO, Green DR: Microtubule-associated protein 1 light chain 3 alpha (LC3)-associated phagocytosis is required for the efficient clearance of dead cells. Proc Natl Acad Sci USA 2011, 108(42):17396-17401.

26. Nakamagoe K, Shioya A, Yamaguchi T, Takahashi H, Koide R, Monzen T, Satoh J, Tamaoka A: A Japanese case with Nasu-Hakola disease of DAP12 gene mutation exhibiting precuneus hypoperfusion. Intern Med 2011, 50(22):2839-2844.

27. Yagishita S, Ito Y, Sakai H, Amano N: Membranocystic lesions of the lung in Nasu-Hakola disease. Virchows Arch A Pathol Anat Histopathol 1985, 408(2-3):211-217.

28. Motohashi N, Shinohara M, Shioe K, Fukuzawa H, Akiyama Y, Kariya T: A case of membranous lipodystrophy (Nasu-Hakola disease) with unique MRI findings. Neuroradiology 1995, 37(7):549-550.

29. Kuhlmann T, Remington L, Maruschak B, Owens T, Brück W: Nogo-A is a reliable oligodendroglial marker in adult human and mouse CNS and in demyelinated lesions. J Neuropathol Exp Neurol 2007, 66(3):238-246.

30. Ohtani K, Suzumura A, Sawada M, Marunouchi T, Nakashima I, Takahashi A Establishment of mouse oligodendrocyte/type-2 astrocyte lineage cell line by transfection with origin-defective simian virus 40 DNA. Cell Struct Funct 1992, 17(5):325-333.

31. Lee Y, Morrison BM, Li Y, Lengacher S, Farah MH, Hoffman PN, Liu Y, Tsingalia A, Jin L, Zhang PW, Pellerin L, Magistretti PJ, Rothstein JD: Oligodendroglia metabolically support axons and contribute to neurodegeneration. Nature 2012, 487(7408):443-448.

32. Lucchinetti CF, Popescu BF, Bunyan RF, Moll NM, Roemer SF, Lassmann H, Brück W, Parisi JE, Scheithauer BW, Giannini C, Weigand SD, Mandrekar J, Ransohoff RM: Inflammatory cortical demyelination in early multiple sclerosis. N Engl J Med 2011, 365(23):2188-2197

33. Aoki N, Tsuchiya K, Togo T, Kobayashi Z, Uchikado H, Katsuse O, Suzuki K, Fujishiro H, Arai T, Iseki E, Anno M, Kosaka K, Akiyama H, Hirayasu Y: Gray matter lesions in Nasu-Hakola disease: a report on three autopsy cases. Neuropathology 2011, 31(2):135-143

34. Lee KM, Hwang SK, Lee JA: Neuronal autophagy and neurodevelopmental disorders. Exp Neurobiol 2013, 22(3):133-142.

35. Numasawa Y, Yamaura C, Ishihara S, Shintani S, Yamazaki M, Tabunoki H, Satoh JI: Nasu-Hakola disease with a splicing mutation of TREM2 in a Japanese family. Eur J Neurol 2011, 18(9):1179-1183.

36. Behrends C, Sowa ME, Gygi SP, Harper JW: Network organization of the human autophagy system. Nature 2010, 466(7302):68-76.

37. Ding Q, Dimayuga E, Martin S, Bruce-Keller AJ, Nukala V, Cuervo AM, Keller JN: Characterization of chronic low-level proteasome inhibition on neural homeostasis. J Neurochem 2003, 86(2):489-497.

38. Knævelsrud $H$, Simonsen A: Fighting disease by selective autophagy of aggregate-prone proteins. FEBS Lett 2010, 584(12):2635-2645.

39. Rothenberg C, Srinivasan D, Mah L, Kaushik S, Peterhoff CM, Ugolino J, Fang S, Cuervo AM, Nixon RA, Monteiro MJ: Ubiquilin functions in autophagy and is degraded by chaperone-mediated autophagy. Hum Mol Genet 2010, 19(16):3219-3232.

40. Deretic $V$, Saitoh T, Akira S: Autophagy in infection, inflammation and immunity. Nat Rev Immunol 2013, 13(10):722-737.

41. Kawaguchi Y, Kovacs JJ, McLaurin A, Vance JM, Ito A, Yao TP: The deacetylase HDAC6 regulates aggresome formation and cell viability in response to misfolded protein stress. Cell 2003, 115(6):727-738.

42. Heir R, Ablasou C, Dumontier E, Elliott M, Fagotto-Kaufmann C, Bedford FK: The UBL domain of PLIC-1 regulates aggresome formation. EMBO Rep 2006, 7(12):1252-1258

43. Komatsu M, Waguri S, Koike M, Sou YS, Ueno T, Hara T, Mizushima N, Iwata J, Ezaki J, Murata S, Hamazaki J, Nishito Y, lemura S, Natsume T, Yanagawa T, Uwayama J, Warabi E, Yoshida H, Ishii T, Kobayashi A, Yamamoto M, Yue Z Uchiyama Y, Kominami E, Tanaka K: Homeostatic levels of p62 control cytoplasmic inclusion body formation in autophagy-deficient mice. Cell 2007, 131(6):1149-1163.

44. Smith CM, Mayer JA, Duncan ID: Autophagy promotes oligodendrocyte survival and function following dysmyelination in a long-lived myelin mutant. J Neurosci 2013, 33(18):8088-8100.

45. Sciarretta S, Hariharan N, Monden Y, Zablocki D, Sadoshima J: Is autophagy in response to ischemia and reperfusion protective or detrimental for the heart? Pediatr Cardiol 2011, 32(3):275-281

46. Jia L, Dourmashkin RR, Allen PD, Gray AB, Newland AC, Kelsey SM: Inhibition of autophagy abrogates tumour necrosis factor alpha induced 
apoptosis in human T-lymphoblastic leukaemic cells. Br J Haematol 1997, 98(3):673-685.

47. Xue L, Fletcher GC, Tolkovsky AM: Autophagy is activated by apoptotic signalling in sympathetic neurons: an alternative mechanism of death execution. Mol Cell Neurosci 1999, 14(3):180-198.

48. Pattingre S, Tassa A, Qu X, Garuti R, Liang XH, Mizushima N, Packer M, Schneider MD, Levine B: BCl-2 antiapoptotic proteins inhibit Beclin 1-dependent autophagy. Cell 2005, 122(6):927-939.

49. Caccamo A, Majumder S, Richardson A, Strong R, Oddo S: Molecular interplay between mammalian target of rapamycin (mTOR), amyloid-b, and Tau: effects on cognitive impairments. J Biol Chem 2010, 285(17):13107-13120.

50. Wang IF, Guo BS, Liu YC, Wu CC, Yang CH, Tsai KJ, Shen CK: Autophagy activators rescue and alleviate pathogenesis of a mouse model with proteinopathies of the TAR DNA-binding protein 43. Proc Natl Acad Sci USA 2012, 109(37):15024-15029.

51. Tyler WA, Gangoli N, Gokina P, Kim HA, Covey M, Levison SW, Wood TL: Activation of the mammalian target of rapamycin (mTOR) is essential for oligodendrocyte differentiation. J Neurosci 2009, 29(19):6367-6378.

doi:10.1186/1750-1172-9-68

Cite this article as: Satoh et al.: LC3, an autophagosome marker, is expressed on oligodendrocytes in Nasu-Hakola disease brains. Orphanet Journal of Rare Diseases 2014 9:68.

\section{Submit your next manuscript to BioMed Central and take full advantage of:}

- Convenient online submission

- Thorough peer review

- No space constraints or color figure charges

- Immediate publication on acceptance

- Inclusion in PubMed, CAS, Scopus and Google Scholar

- Research which is freely available for redistribution 